\title{
UMA PROPOSTA DE INTEGRAÇÃO DO TEMA GERADOR À ESTRUTURA CURRICULAR TRANSDISCIPLINAR PARA O ENSINO PROFISSIONAL
}

Reinaldo Golmia Dante - golmia@ifsp.edu.br

Instituto Federal de Educação, Ciência e Tecnologia de São Paulo - Câmpus Sertãozinho, Coordenadoria de Elétrica e Computação

Rua Américo Ambrósio, 269

CEP: 14169-263 - Sertãozinho - SP

Resumo: Diante das mudanças e necessidades da sociedade neste século XXI, novos desafios em todos os campos de atuação têm sido impostos às pessoas, exigindo que desenvolvam maior capacidade de entendimento da realidade e análise dos fatos. Nesse sentido, uma visão sistêmica desse universo poderia ser alcançada, por exemplo, por meio de uma formação acadêmica aberta ao mundo exterior que proporcione a compreensão das diversas áreas do conhecimento e suas inter-relações, objetivando a proximidade de se visualizar o conhecimento como sendo uno. $O$ pensamento complexo crítico e a transdisciplinaridade como base de um Currículo constituem um caminho confiável para a aquisição dessa visão sistêmica. O tema gerador é essencial em uma estrutura curricular transdisciplinar para alinhar todas as ações em direção a uma finalidade comum dentro de um sistema educacional. Este trabalho tem por objetivo apresentar um estudo teórico de cunho prático sobre como o tema gerador poderia ser integrado a uma estrutura curricular transdisciplinar para o Ensino Profissional, o qual contempla o Ensino Técnico de nível médio e o superior.

Palavras-chave: Tema gerador. Estrutura curricular. Transdisciplinaridade. Pensamento complexo crítico. 


\section{INTRODUÇÃO}

Os desafios do século XXI, manifestados nos inúmeros campos, tais como: do Meio Ambiente, da Economia, do Desenvolvimento Social e outros, têm exigido do ser humano uma capacidade de raciocínio e análise cada vez mais complexa em virtude da tendência de unificação do conhecimento que outrora foi particionado e isolado pela vontade do próprio ser humano em tornar cada parte especializada. A Eletrônica e o Direito apresentam muitos pontos em comum como os contratos eletrônicos, a investigação de crimes cibernéticos, e a própria petição inicial e os recursos são digitais. A Engenharia Biomédica é um exemplo de convergência entre os propósitos da Engenharia e da Medicina. Atualmente, não é mais possível aspirar por avanços tecnológicos sem considerar os seus impactos ambientais, econômicos, sociais etc. Todos esses exemplos evidenciam que é preciso mudar o modo de pensar para melhor compreender a realidade deste mundo.

Nesse sentido, a Educação precisa estar atenta a essas mudanças e necessidades da sociedade e promover uma formação cidadã e acadêmica alinhadas a elas. Para isso, exige-se que o pensamento complexo crítico e a transdisciplinaridade estejam intimamente relacionados com o Currículo para proporcionar ao educando uma visão sistêmica da realidade, entendendo-a em seus diversos níveis.

Este trabalho tem por objetivo apresentar uma proposta de integração do tema gerador à estrutura curricular transdisciplinar para o Ensino Profissional que será discutido nas seções seguintes.

\section{FUNDAMENTAÇÃO TEÓRICA}

As bases teóricas da Educação sobre os conceitos de disciplinaridade, multidisciplinaridade, pluridisciplinaridade, interdisciplinaridade e transdisciplinaridade são apresentadas nesta seção. A disciplinaridade pode ser entendida dentro de um sistema como uma disciplina isolada e especializada em um determinado domínio homogêneo de uma parte do conhecimento (ex.: Inglês) sem qualquer cooperação ou mesmo coordenação. Baseado no trabalho de Jantsch (1972 apud JAPIASSU, 1976, p. 73-74), Japiassu descreve a multidisciplinaridade como uma "gama de disciplinas que propomos simultaneamente, mas sem fazer aparecer as relações que podem existir entre elas" (JAPIASSU, 1976, p. 73). Nesse sentido, a multidisciplinaridade é um conjunto de disciplinas isoladas (ex.: Inglês, Matemática, Eletrônica Digital etc.), sendo que cada uma possui uma especialização em um determinado conhecimento. Em termos sistêmicos, a multidisciplinaridade pode ser considerada como um único nível, em que as disciplinas estejam alinhadas horizontalmente sob esse patamar, e contém múltiplos objetivos decorrentes desse conjunto disciplinar, porém ainda sem qualquer cooperação ou coordenação. Tomando-se, como exemplo, uma equipe multidisciplinar de pesquisadores, cada um apresenta a sua própria análise separadamente sob uma perspectiva individualizada e restrita à sua área de conhecimento (MAX-NEEF, 2005, p. 6). A pluridisciplinaridade pode ser considerada como um aperfeiçoamento do sistema multidisciplinar, pois, ao passo que aquela não possui qualquer cooperação ou coordenação, esta apresenta relações de cooperação, mas não de coordenação, entre as disciplinas ainda que todas estejam situadas no mesmo nível. No sistema pluridisciplinar, é possível estabelecer relações entre conteúdos das diversas disciplinas que o compõe e explorar suas afinidades. Considerando, por exemplo, as disciplinas de Inglês, Matemática e Eletrônica Digital, buscase verificar quais conteúdos de cada uma delas podem ser explorados com o objetivo de auxiliar a melhor compreensão de outra disciplina. Nesse sentido, o Inglês poderá auxiliar a compreensão da Eletrônica Digital por meio dos termos técnicos internacionais, os quais são 
padronizados no idioma inglês; a Matemática proverá, por exemplo, o conhecimento de Álgebra que auxiliará o entendimento de como projetar circuitos lógicos em Eletrônica Digital; e, por fim, os dispositivos eletrônicos (ex: computadores, projetores multimídia) da Eletrônica Digital poderão ser empregados para auxiliar na compreensão dos conteúdos das disciplinas de Inglês e Matemática. A interdisciplinaridade apresenta um grau de complexidade maior que os sistemas anteriores, pois ela opera em dois níveis hierárquicos: no plano inferior, situam-se as disciplinas e suas relações de cooperação análogas ao sistema pluridisciplinar; e no plano superior, uma axiomática comum estabelece uma relação de coordenação sob esse conjunto de disciplinas conexas (JAPIASSU, 1976; MAX-NEEF, 2005). Japiassu (1976, p. 74) descreve esse nível imediatamente superior como "o que introduz a noção de finalidade". O sistema interdisciplinar pode ser, portanto, visualizado como um modelo piramidal reduzido a dois níveis, cuja base representa um conjunto disciplinar e o topo a coordenação motivada pela finalidade. Max-Neef (2005, p. 7 e 8) salienta ainda que esse modelo piramidal pode ocorrer em quatro diferentes níveis hierárquicos interdisciplinares: a) o primeiro nível é o empírico - a base dessa pirâmide - que contempla todas as disciplinas, tais como: Química, Biologia, Sociologia, Solo etc.; b) o segundo nível é o pragmático que reúne um outro grupo de elementos, os quais visam estabelecer algumas relações de coordenação; nesse caso, cita-se a Agricultura, capaz de coordenar as relações entre a Química, Biologia, Sociologia e Solo; c) o terceiro nível é o normativo que estabelece ações de coordenação, por meio dos elementos normativos (ex.: Política, Planejamento etc.), entre os elementos do nível pragmático; e d) o último nível, topo dessa pirâmide, é o de valores que engloba um pequeno grupo de elementos, tais como: Ética e Filosofia, e tem por objetivo coordenar ações entre os elementos normativos. No entanto, apesar de esse sistema interdisciplinar permitir explorar quatro níveis hierárquicos, eles estão dissociados, ou seja, o sistema interdisciplinar pode ser compreendido como um conjunto de três pirâmides autônomas de dois níveis: a primeira, engloba os níveis empírico e pragmático; a segunda, os níveis pragmático e normativo; e a terceira, os níveis normativo e de valores. Não há inter-relação entre essas pirâmides e, consequentemente, não é possível identificar uma finalidade comum para todo o sistema. Essa inter-relação é alcançada no sistema transdisciplinar. A transdisciplinaridade permite que haja coordenação de todos os elementos de um sistema por meio dessa inter-relação entre os níveis hierárquicos interdisciplinares a fim de alcançar um propósito convergente para o sistema. Portanto, o sistema transdisciplinar apresenta o maior grau de complexidade comparado aos demais sistemas supramencionados.

Sob a perspectiva do contexto histórico, os três grandes pensadores Jean Piaget, Erich Jantsch e André Lichnerowicz foram os primeiros a mencionar o termo transdisciplinaridade em seus discursos, ainda que essa terminologia não fosse consensual, na oficina internacional Interdisciplinaridade: problemas de ensino e de pesquisa nas universidades organizada pela Organização para Economia, Cooperação e Desenvolvimento (OECD), na França, em 1970. Provavelmente, a comunidade acadêmica daquela época não estava preparada para receber uma proposta inovadora e ousada que pudesse romper as fronteiras existentes das disciplinas e de suas relações (i.e., pluridisciplinaridade e interdisciplinaridade), provendo esperança de explorar um novo campo do conhecimento (NICOLESCU, 2005). Piaget (1972, p. 170) afirmou que a transdisciplinaridade, como um estágio superior à interdisciplinaridade, não se contenta em apenas alcançar as relações de cooperação e coordenação entre as disciplinas, mas em situá-las no interior de um sistema total sem fronteiras estáveis entre elas. Em termos de uma visão sistêmica, Jantsch (1970, p. 16) reiterou o entendimento de que a transdisciplinaridade é a "coordenação de todas as disciplinas e interdisciplinas" e propôs uma estrutura universitária transdisciplinar baseada em seu modelo hierárquico. A base teórica da transdisciplinaridade não estava completa até a propositura de uma formulação axiomática da 


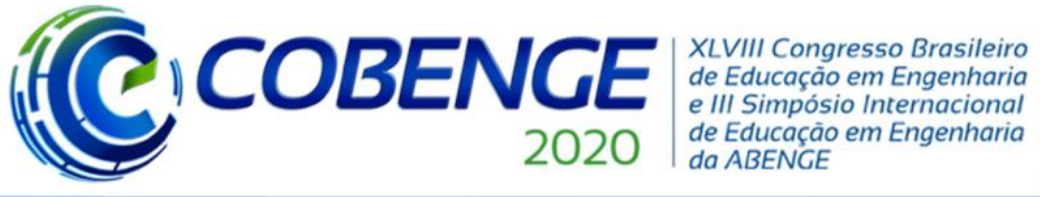

"Os desafios para formar hoje o engenheiro do amanhã"

metodologia de transdisciplinaridade por Nicolescu (2005, p. 5-14) que preencheu essa lacuna.

A partir dessa formulação, a transdisciplinaridade pôde alcançar uma questão filosófica sobre “O que é a Realidade? Há relação entre a Realidade e a Percepção?” e, em seu bojo axiomático, encontra-se o axioma ontológico, cuja definição é "Há, na Natureza e em nosso conhecimento da Natureza, diferentes níveis de Realidade e, correspondentemente, diferentes níveis de Percepção" (NICOLESCU, 2005, p. 6, tradução minha). Ter ciência de que a realidade exibe diversos níveis e cada um deles está associado a um nível de percepção, além de proporcionar uma amplificação do conhecimento de universo, permite à estrutura curricular que ela seja construída de uma forma mais realista e contemporânea, atenta às mudanças e necessidades da sociedade que este Currículo se propõe. Nesse sentido, é imprescindível que seja dada primazia ao universo exterior quanto ao olhar que essa estrutura curricular deva objetivar, pois a Realidade é complexa em sua natureza, e é por meio do pensamento complexo crítico em consonância com a transdisciplinaridade que se possa vislumbrar um caminho que leve à construção de um Currículo adequado ao educando para enfrentar os seus desafios futuros. Diante desse cenário, a escola deve estimular o educando a adquirir a consciência da realidade, pois ela conduz à reflexão crítica dos ambientes físico, econômico, social, educacional dentre outros, incluindo as suas inter-relações, que ele está inserido. Ademais, o pensamento complexo crítico e a transdisciplinaridade propiciam ao educando algumas vantagens preciosas: a visão sistêmica, a contextualização e a significação.

Contrária à política reducionista do conhecimento, a qual busca o entendimento de um fenômeno mediante a simplificação do número de variáveis e condições que o incidem, a visão sistêmica, situada em um plano superior, permite aproximar à compreensão integrada desse fenômeno ao ambiente, incluindo-se, para isso, as suas inter-relações abertas, além de assegurar a expansão desse conhecimento. $\mathrm{O}$ entendimento da dinâmica desse fenômeno no universo exterior está intimamente relacionado com a contextualização e significação de conceitos ali presentes. Exemplificando, para entender o fenômeno pandemia da Covid-19, é necessário considerar a sua origem e os multifatores biológico, cultural, social, econômico, educacional, tecnológico dentre outros (inter-relações abertas). De acordo com informações da Organização Mundial da Saúde (OMS), os primeiros casos de pneumonia causados por um novo tipo de coronavírus, chamado de SARS-CoV-2, surgiram em Wuhan, China, em 31 de dezembro de 2019 e, desde então, a Covid-19 se espalhou para a Ásia, Europa e demais continentes, sendo decretada a situação de pandemia em 11 de março de 2020 (WORLD HEALTH ORGANIZATION, 2020). Quanto à sua origem, suspeita-se que esse vírus seja proveniente de morcegos e/ou pangolins, pois alguns tipos de coronavírus, os quais se aproximam ao SARS-CoV-2, foram encontrados nesses animais, e alguns focos preliminares foram encontrados em mercados de vendas de animais selvagens (fator cultural), o que leva a entender que os primeiros contágios tenham ocorrido naquele local (BRITISH BROADCASTING CORPORATION, 2020). Em termos biológicos, o coronavírus SARSCoV-2 ataca diversos órgãos do corpo humano, em especial, os pulmões, e transmissão ocorre pela via aérea ou ainda pelo contato físico ou de objetos contaminados. A maior probabilidade de propagação dessa doença ocorre em locais com aglomeração de pessoas como, por exemplo, os grandes centros urbanos (fator social). Em termos econômicos, a pandemia não só causou enormes prejuízos nos países como também encontrou caminhos para sua expansão via comunidades carentes em virtude das baixas taxas de saneamento básico e altas concentrações de pessoas. A falta de cuidados básicos de higiene (ex.: deixar de lavar as mãos frequentemente, as roupas e os sapatos ao chegar em casa) contribui para a disseminação dessa doença (fator educacional). Como fator tecnológico, a escassez de Equipamentos Protetores Individuais (EPIs) e as falhas na divulgação de informações assertivas de 
prevenção (ex.: notícias falsas) às pessoas pelos meios de comunicação podem contribuir para a expansão da Covid-19. Ademais, além daqueles fatores supramencionados, deve-se analisar também as suas inter-relações existentes como, por exemplo, deixar de lavar as mãos por falta de saneamento básico. Observe, portanto, que a compreensão do fenômeno pandemia da Covid-19 se aproxima cada vez mais da realidade quanto maior for a discriminação de fatores e suas interconexões a serem examinadas de forma crítica.

Reflexões dessa natureza são fundamentais para a construção de um projeto pedagógico curricular calcado no pensamento complexo crítico e na transdisciplinaridade sem se esquecer, no entanto, da importância das disciplinas nesse processo. A transdisciplinaridade não conflita com nenhuma outra categoria anterior, aliás, ela é complementar às demais. Uma premissa relevante a respeito da transdisciplinaridade é de que ela não existiria se não houvesse a disciplinaridade (NICOLESCU, 2005, p. 4). Deve-se lembrar que a transdisciplinaridade estabelece relações de coordenação entre as disciplinas em diferentes níveis (inter-relação entre os planos interdisciplinares) dentro de um sistema. Assim, apesar de as fronteiras não serem estáveis, isso ocorre no sentido de assegurar um fortalecimento e maior cooperação entre essas disciplinas, mas elas continuam existindo (JANTSCH, 1970; PIAGET, 1972).

Apoiando-se em Macedo (2008) e Moraes (2008), Moraes (2010, p. 15) afirma que

Um currículo transdisciplinar, nutrido pela complexidade, trabalha com projetos, com perguntas mediadoras, com questões temáticas e problemas, com ciclos de aprendizagem, trabalha também com sínteses integradoras, com relações contextuais, históricas e políticas, sínteses estas requeridas pela problemática humana e pelos seus desafios.

Esses elementos apontados por Moraes (2010) representam algumas ideias que podem auxiliar a implementação de um currículo transdisciplinar.

Quanto à abordagem desse assunto por meio dos documentos oficiais do Ministério da Educação (MEC), observa-se que, apesar de haver uma preocupação em aproximar o Currículo à formação dos estudantes direcionada às necessidades da sociedade, a transdisciplinaridade ainda é um tema incipiente e muito pouco explorada, reflexo da lentidão em se materializar como política pública pelo MEC. Nas orientações, tanto curriculares para o Ensino Médio quanto educacionais complementares aos Parâmetros Curriculares Nacionais, discute-se a importância dos temas transversais como caminhos motivadores para o aprimoramento do processo de ensino-aprendizagem por meio da articulação entre os conteúdos das diversas disciplinas (BRASIL, 2002, 2006). Entende-se que esse foi o primeiro estágio rumo à transdisciplinaridade. Transcorrida uma década, introduziram-se, como princípios da Educação Profissional Técnica de Nível Médio, a contextualização, a flexibilidade e a interdisciplinaridade nos termos do artigo $6^{\circ}$, inciso VIII, da Resolução n. 6 , de 20 de setembro de 2012 (BRASIL, 2012). Finalmente, após seis anos, a transdisciplinaridade é mencionada, porém uma única vez, na Base Nacional Curricular Comum (BNCC) dos Ensinos Fundamental e Médio (BRASIL, 2018a, p. 479) ao fazer referência ao artigo $7^{\circ}, \S 2^{\circ}$, da Resolução n. 3, de 21 de novembro de 2018 , que prescreve "O currículo deve contemplar tratamento metodológico que evidencie a contextualização, a diversificação e a transdisciplinaridade ou outras formas de interação e articulação entre diferentes campos de saberes específicos (...)" (BRASIL, 2018b, p. 24, grifo meu).

Infelizmente, a transdisciplinaridade foi tratada "en passant" na BNCC, a qual se limitou a apresentá-la como "uma das formas de interação e articulação entre diferentes campos de saber específicos" (BRASIL, 2018a, p. 479) sem que houvesse uma conceituação a seu respeito. A Resolução n. 3, de 21 de novembro de 2018, abordou com maior profundidade maior a relação entre a transdisciplinaridade e o Currículo, a exemplo, o seu artigo $11, \S 2^{\circ}$, 
estabeleceu que "O currículo por área de conhecimento deve ser organizado e planejado dentro das áreas de forma interdisciplinar e transdisciplinar" (BRASIL, 2018b, p. 26). Constata-se, portanto, que a organização curricular dar-se-á por áreas de conhecimento, já prevista nos Parâmetros Curriculares Nacionais para o Ensino Médio (PCNEM), a saber: "Linguagens, Códigos e suas Tecnologias, Ciências da Natureza, Matemática e suas Tecnologias e Ciências Humanas e suas Tecnologias" (BRASIL, 2000, p. 18), e que o diálogo entre essas áreas ocorrerá na forma mencionada anteriormente.

Quanto à relação entre a transdisciplinaridade o Currículo nas normas oficiais dos cursos superiores, por exemplo, a Resolução ${ }^{\circ}$ 2, de 24 de abril de 2019, que institui as Diretrizes Curriculares Nacionais do Curso de Graduação em Engenharia, em seu Capítulo III - Da Organização do Curso de Graduação em Engenharia, mais especificamente, no artigo $6^{\circ}, \S 8^{\circ}$, prescreve que "Devem ser estimuladas as atividades acadêmicas, tais como trabalhos de iniciação científica, competições acadêmicas, projetos interdisciplinares e transdisciplinares, projetos de extensão (...)" (BRASIL, 2019, p. 46, grifo meu).

Observa-se que, nessa resolução, a transdisciplinaridade, infelizmente, foi reduzida, em sua potencialidade, ao patamar de atividade acadêmica, em particular, projetos. Conforme mencionado anteriormente, a transdisciplinaridade propõe a transposição de barreiras entre as disciplinas dentro de um sistema, o que permite ampliar o conhecimento em busca de uma visão sistêmica e melhor compreensão da Realidade e seus diversos níveis. Ademais, todos os meios de implementação de um currículo transdisciplinar deveriam ser considerados nessa norma a fim de manter esse rol em aberto para que não se torne taxativo.

Por fim, alguns estudos sobre estruturas curriculares transdisciplinares foram propostos (DANTE et al., 2019; JANTSCH, 1970; MAX-NEEF, 2005; QUINTERO, 2005). Este trabalho discutirá a proposta de uma possível integração do tema gerador ao modelo de estrutura curricular transdisciplinar proposto por Dante et al. (2019) para o Ensino Profissional.

\section{METODOLOGIA}

Este trabalho é fruto de uma análise teórica de cunho prático baseada em pesquisas bibliográficas sobre o pensamento complexo, a transdisciplinaridade e outras categorias organizacionais curriculares, bem como as suas relações com o Currículo, incluindo o levantamento de documentos oficiais publicados pelo MEC para os Ensinos Técnico/Médio e Superior de Graduação de Engenharia, além da organização dos textos e compilação desses estudos.

\section{RESULTADO E DISCUSSÕES}

Inicialmente, faz-se necessário traçar algumas considerações e premissas sobre a estrutura curricular proposta por Dante et al. (2019). O Ensino Profissional é compreendido pelos Ensinos Técnico de nível médio e o Superior. São modalidades dos cursos técnicos de nível médio os cursos integrados ao Ensino Médio (i.e., disciplinas técnicas e científicas integradas em uma única Instituição), Educação de Jovens e Adultos Profissionalizante (PROEJA), concomitantes (i.e., quando o estudante cursa, separadamente, as disciplinas técnicas e científicas em diferentes Instituições simultaneamente) e subsequente (i.e., os estudantes, portadores de diploma de Ensino Médio, cursam somente as disciplinas técnicas). São modalidades dos cursos superiores os cursos de graduação em Tecnologia e Engenharia. Segundo Dante et al. (2019, p. 5): 
Nessa proposta, a transdisciplinaridade ocorre de modo intracursos e intercursos. No modo intracursos, a transdisciplinaridade permeia em cada curso por meio do diálogo entre as diversas áreas do conhecimento (e.g., Exatas, Eletrônica) alinhadas às dimensões (e.g., Economia, Meio Ambiente) que promovem diretrizes fenomenológicas a serem refletidas pela comunidade escolar (e.g., estudantes, docentes, técnicos-administrativos). No modo intercursos, a transdisciplinaridade permeia entre um curso de nível médio e outro de nível superior de modo que haverá um maior engajamento entre esses estudantes de diferentes níveis na participação das atividades transdisciplinares (e.g., projetos, problematizações).

Trata-se, portanto, de admissibilidade dessa estrutura curricular para a transdisciplinaridade nas formas horizontal e vertical. Ademais, destaca-se que as disciplinas deverão ser mantidas para que haja a transdisciplinaridade, conforme já exposto anteriormente.

No que se refere ao tema gerador, a Resolução n. 3, de 21 de novembro de 2018, artigo $17, \S 7^{\circ}$, preconiza

As áreas do conhecimento podem ser organizadas em unidades curriculares, competências e habilidades, unidades de estudo, módulos, atividades, práticas e projetos contextualizados ou diversamente articuladores de saberes, desenvolvimento transversal ou transdisciplinar de temas ou outras formas de organização (BRASIL, 2018b, p. 30, grifo meu).

Nota-se que, nessa norma, foi contemplada um rol aberto de alternativas para viabilizar a transdisciplinaridade no Currículo, diferentemente da Resolução no 2 , de 24 de abril de 2019, que optou pela restrição estrita, apontado em seu artigo $6^{\circ}, \S 8^{\circ}$, aos projetos transdisciplinares.

Explorar a transdisciplinaridade por meio de temas geradores torna as relações de coordenação e cooperação entre as disciplinas dentro de um sistema mais amplas e enriquecidas, pois a partir de um tema gerador, pode-se florescer vários projetos. Por essa razão, propõe-se, neste trabalho, que o tema gerador esteja situado em um plano hierárquico superior aos cursos e transpasse todas as dimensões, conforme é ilustrado na Figura 1.

Figura 1 - Integração do tema gerador à estrutura curricular transdisciplinar.

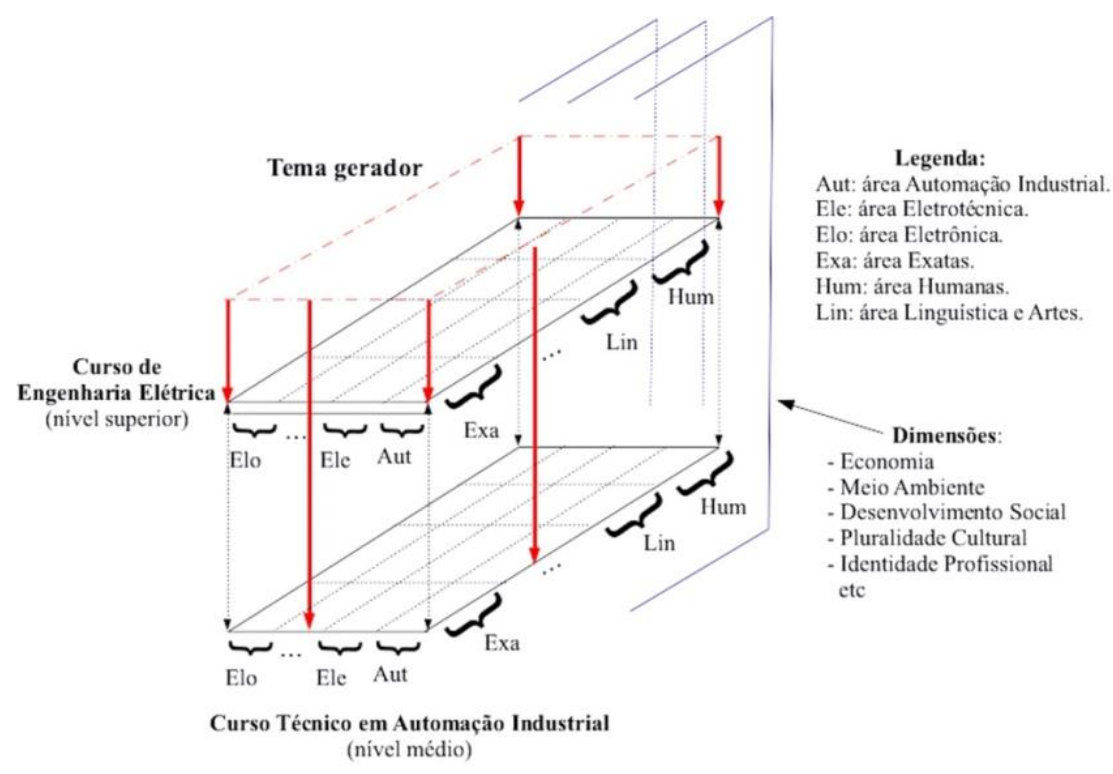

Fonte: adaptação de Dante et al. (2019, p. 6). 
O tema gerador incide sobre todos os cursos e norteia as suas ações, direcionando-os para uma finalidade comum para todo o sistema. Retomando o exemplo sobre o fenômeno pandemia da Covid-19, sua origem e os multifatores biológico, cultural, social, econômico, educacional, tecnológico dentre outros (inter-relações abertas), identifica-se o tema gerador como sendo a pandemia da Covid-19. É importante identificar também o núcleo do tema gerador, que nesse caso, é a Covid-19, pois com base nele, outros temas geradores correlatos são possíveis de serem formulados, como, por exemplo, a prevenção contra a Covid-19. Ademais, impulsionado pelo tema gerador, pode-se ainda propor questionamentos, problematizações e/ou projetos. No que se refere ao tema gerador pandemia da Covid-19, sugere-se formular, por exemplo, o questionamento "Como os Estados Unidos, a maior economia mundial, pôde perder, de certo modo, o controle da disseminação da Covid-19, se comparado com outros países, por atingir o número recorde de óbitos?". Um projeto sobre a análise geoeconômica associada às políticas públicas de saúde dos Estados Unidos durante a pandemia da Covid-19 poderia também ser oferecido. Para esse exemplo, as dimensões da estrutura curricular transdisciplinar se aproximam daqueles multifatores, os quais auxiliaram a tornar mais realista a compreensão do fenômeno. Com relação ao tema gerador prevenção contra a Covid-19, alguns projetos técnicos, como as construções de EPIs e aparelhos de respiração eletromecânica e a produção de álcool em gel, seriam ótimas ideias para engajar os estudantes dos cursos superiores (ex.: Química, Engenharias Elétrica e Mecatrônica) e técnicos (ex.: Química, Eletrônica e Automação Industrial). As dimensões, para esse caso, poderiam ser as mesmas apresentadas na Figura 1, pois a construção de um aparelho respiratório eletromecânico perpassa as perspectivas econômicas, ambientais dentre outras.

Por fim, conforme o exposto, fica manifestada a viabilidade dessa proposta de integração do tema gerador à estrutura curricular transdisciplinar apresentado por Dante et al. (2019).

\section{CONSIDERAÇÕES FINAIS}

Esse trabalho apresentou um estudo de uma possível forma de integração do tema gerador à estrutura curricular transdisciplinar apresentada por Dante et al. (2019) para o Ensino Profissional. Essa proposta se mostra factível de ser implementada na prática por permitir que os temas geradores promovam a convergência de todas as ações norteadas pelas relações de coordenação e cooperação para construção de uma finalidade comum delineada nos Planos Pedagógicos de Cursos.

\section{REFERÊNCIAS}

BRASIL. Ministério da Educação (MEC). Secretaria de Educação Básica (SEB). Parâmetros Curriculares Nacionais para o Ensino Médio: Parte I - Bases Legais. Brasília: SEB/MEC, 2000.

. Ministério da Educação (MEC). Secretaria de Educação Básica (SEB). PCN+ Ensino Médio: orientações educacionais complementares aos Parâmetros Curriculares Nacionais: ciências da natureza, matemática e suas tecnologias. Brasília: SEB/MEC, 2002.

Ministério da Educação (MEC). Secretaria de Educação Básica (SEB). Orientações

Curriculares para o Ensino Médio: ciências da natureza, matemática e suas tecnologias. V.

2. Brasília: SEB/MEC, 2006. 
Ministério da Educação (MEC). Conselho Nacional de Educação (CNE). Câmara de Educação Básica. Resolução ${ }^{0}$ 6, de 20 de setembro de 2012. Diário Oficial da União. DF, 21 set. 2012. Seção 1, p. 22-24.

Ministério da Educação (MEC). Secretaria de Educação Básica (SEB). Base Nacional Comum Curricular: Educação é a base. Brasília: SEB/MEC, 2018a.

Ministério da Educação (MEC). Conselho Nacional de Educação (CNE). Câmara de Educação Básica. Resolução $n^{0}$ 3, de 21 de novembro de 2018. Diário Oficial da União. DF, 22 nov. 2018b. Seção 1, p. 21-37.

Ministério da Educação (MEC). Conselho Nacional de Educação (CNE). Câmara de Educação Superior. Resolução $\mathrm{n}^{\circ}$ 2, de 24 de abril de 2019. Diário Oficial da União. DF, 26 abr. 2019. Seção 1, p. 43-49.

BRITISH BROADCASTING CORPORATION. Coronavirus: Pangolins found to carry related strains. BBC Science \& Environment, mar., 2020. Disponível em:

https://www.bbc.com/news/science-environment-52048195. Acesso em: 08 jul. 2020.

DANTE, R. G. et al. Proposta de uma estrutura curricular transdisciplinar para o Ensino Profissional. In: VII Congresso Brasileiro de Educação (CBE): "Educação pública como direito: desafios e perspectivas no Brasil contemporâneo", 2019. Anais. Bauru, 2019. Disponível em: https://cbe-unesp.com.br/anais/index.php?t=RE2019215099948. Acesso em: 08 jul. 2020.

JANTSCH, E. Inter- and transdisciplinary university: a systems approach to education and innovation. v. 1, Issue 1, p.403-428, Springer Policy Sciences, 1970.

JAPIASSU, Hilton. Interdisciplinaridade e patologia do saber. Rio de Janeiro: IMAGO, 1976.

MAX-NEEF, M. Foundations of transdisciplinarity [Commentary]. Ecological Economics, v. 53, p. 5-16, 2005.

MACEDO, Roberto Sidnei. Currículo: campo, conceito e pesquisa. Petrópolis: Vozes, 2008.

MORAES, Maria Cândida. Ecologia dos saberes: Complexidade, transdisciplinaridade e educação. São Paulo: Antakarana/ProLibera, 2008.

Complexidade e currículo: por uma nova relação. Polis Revista Latinoamericana, v. 25, p. 1-20, 2010. Disponível em: http://polis.revues.org/573. Acesso em: 08 jul. 2020.

NICOLESCU, B. Transdisciplinarity - past, present and future. In: Second World Congress of Transdisciplinarity: What Education for Sustainable Development? Attitude - Research Action, 2005. Anais. Vila Velha/Vitória, p. 1-24, 2005. 
PIAGET, J. L'épistémologie des relations interdisciplinaires. In: L'interdisciplinarité: problèmes d'enseignement et de recherche dans les universités, 1972, Paris. Anais eletrônicos... Paris: OCDE, p. 131-144, 1972. Disponível em:

http://www.fondationjeanpiaget.ch/fjp/site/presentation/index.php?DOCID=996. Acesso em: 08 jul. 2020.

QUINTERO, M. A. Three-dimensional model of transversality. Investigación y Postgrado, Caracas, v. 20, n. 2, p. 241-274, 2005. Disponível em:

http://ve.scielo.org/scielo.php?script=sci_arttext\&pid=S1316-

00872005000200009\&lng=en\&nrm=iso. Acesso em: 08 jul. 2020.

WORLD HEALTH ORGANIZATION. WHO Timeline - COVID-19. WHO Newsroom, abr., 2020. Disponível em: https://www.who.int/news-room/detail/27-04-2020-who-timeline--covid-19. Acesso em: 08 jul. 2020.

\title{
A PROPOSAL FOR INTEGRATING THE GENERATOR TOPIC TO THE TRANSDISCIPLINARY CURRICULUM STRUCTURE FOR PROFESSIONAL EDUCATION
}

\begin{abstract}
In view of the changes and needs of society in this 21st century, new challenges in all fields of activity have been imposed on people, requiring them to develop a greater capacity to understand reality and analyse facts. In this sense, a systemic view of this universe could be achieved, for example, through an academic training open to the outside world that provides an understanding of the various areas of knowledge and their interrelations, aiming at the proximity of viewing knowledge as being one. Critical complex thinking and transdisciplinarity as the basis of a Curriculum constitute a reliable way to acquire this systemic view. The generator topic is essential in a transdisciplinary curriculum structure to align all actions towards a common purpose within an educational system. This work aims to present a theoretical study of a practical nature on how the generator topic could be integrated into a transdisciplinary curriculum structure for Professional Education, which contemplates technical education at high school and higher level.
\end{abstract}

Keywords: generator topic, curriculum structure, transdisciplinarity, critical complex thinking. 